\title{
Low-Dose Volume-of-Interest C-Arm CT Imaging of Intracranial Stents and Flow Diverters
}

\author{
(D). Yang, (D)A. Ahmed, (D) S. Schafer, DD. Niemann, (D)B. Aagaard-Kienitz, (D) K. Royalty, and (D). Strother
}

\begin{abstract}
BACKGROUND AND PURPOSE: Volume-of-interest C-arm CT is a novel technique for imaging of intracranial high-contrast objects. We performed this study to evaluate the potential diagnostic value and radiation dose reduction of this technique for imaging of intracranial stents and flow diverters.
\end{abstract}

MATERIALS AND METHODS: Twenty-seven patients were imaged with a VOI C-arm CT scan following treatment with a flow diverter or stent-assisted coiling. The radiation dose-area product was recorded for VOI scans. For comparison, the dose-area product from 30 previously acquired consecutive full-view DynaCTs was used. Thermoluminescence dosimetry by using 35 evenly distributed thermoluminescence dosimeters in an anthropomorphic head phantom was also performed by using both conventional full field and vOl acquisitions. Three observers were presented with VOI images for assessment of the potential diagnostic value.

RESULTS: The dose-area product measurements showed an exposure reduction of $85 \%$ compared with the full field acquisitions used for comparison. The thermoluminescence dosimetry evaluations also showed a considerable dose reduction of $79.8 \%$ throughout the volume. For most of the evaluated cases, the observers thought that diagnostically useful information was provided by the VOI images $(\alpha=.810)$. Visualization of device details, such as the extent of opening, positioning, wall apposition, and aneurysm coverage, was judged of good diagnostic quality for most cases (88.9\%-92.6\%).

CONCLUSIONS: In this study, VOIC-arm CT provided high-quality diagnostic images of intracranial stents and flow diverters at a dramatic reduction of radiation exposure. Image content was thought to add useful information. It is a promising method to assess device status during procedures and at follow-up.

ABBREVIATIONS: $C A C T=C$-arm CT; FD $=$ flow diverter; FFOV $=$ full FOV; $T L D=$ thermoluminescence dosimetry

$F^{2}$ or safe and effective use, careful assessment during and after deployment of intracranial stents and flow diverters is required to ensure full deployment, proper positioning, and full apposition to the vascular wall. These features are important because they may influence further treatment decisions (eg, balloon dilation and anticoagulation during and/or after a procedure). They are also important in predicting postprocedural aneurysm occlusion. Currently, however, clear visualization is

Received May 26, 2015; accepted after revision August 18.

From the Department of Neurosurgery (P.Y.), Changhai Hospital, Second Military Medical University, Shanghai, China; Departments of Radiology (P.Y., C.S.) and Neurological Surgery (A.A., D.N., B.A.-K.), University of Wisconsin School of Medicine and Public Health, Madison, Wisconsin; and Siemens Medical Solutions USA (S.S., K.R.), Hoffman Estates, Illinois.

Please address correspondence to Pengfei Yang, MD, Department of Neurosurgery, Changhai Hospital, Second Military Medical University, Shanghai, China; Department of Radiology, University of Wisconsin-Madison, Madison, Wisconsin; e-mail: 15921196312@163.com, pyang75@wisc.edu

http://dx.doi.org/10.3174/ajnr.A4590 often impossible with standard-projection $\mathrm{x}$-ray imaging and fluoroscopy.

Currently available C-arm CT (CACT) provides high-spatialresolution images of implantable devices. It has been widely used and is accepted as providing such information. ${ }^{1-4}$ The radiation dose required, however, is approximately twice that of a conventional multidetector row $\mathrm{CT}$ of the brain and thus is a limitation of the technique. ${ }^{5}$ This limitation increases in a workflow in which multiple CACT scans are used during a procedure. Several methods are available to reduce the CACT radiation dose. These include $\mathrm{x}$-ray tube current modulation, peak kilovoltage optimization, and $\mathrm{x}$-ray beam collimation. ${ }^{6}$

A different strategy for dose reduction is to reduce the patient exposure in areas of low interest (eg, those away from an implanted device and the related vasculature). Suggested approaches for this strategy range from reducing the dose outside the area of interest by using $\mathrm{x}$-ray beam attenuating techniques or by box collimating to the area of interest. The beam-attenuating 


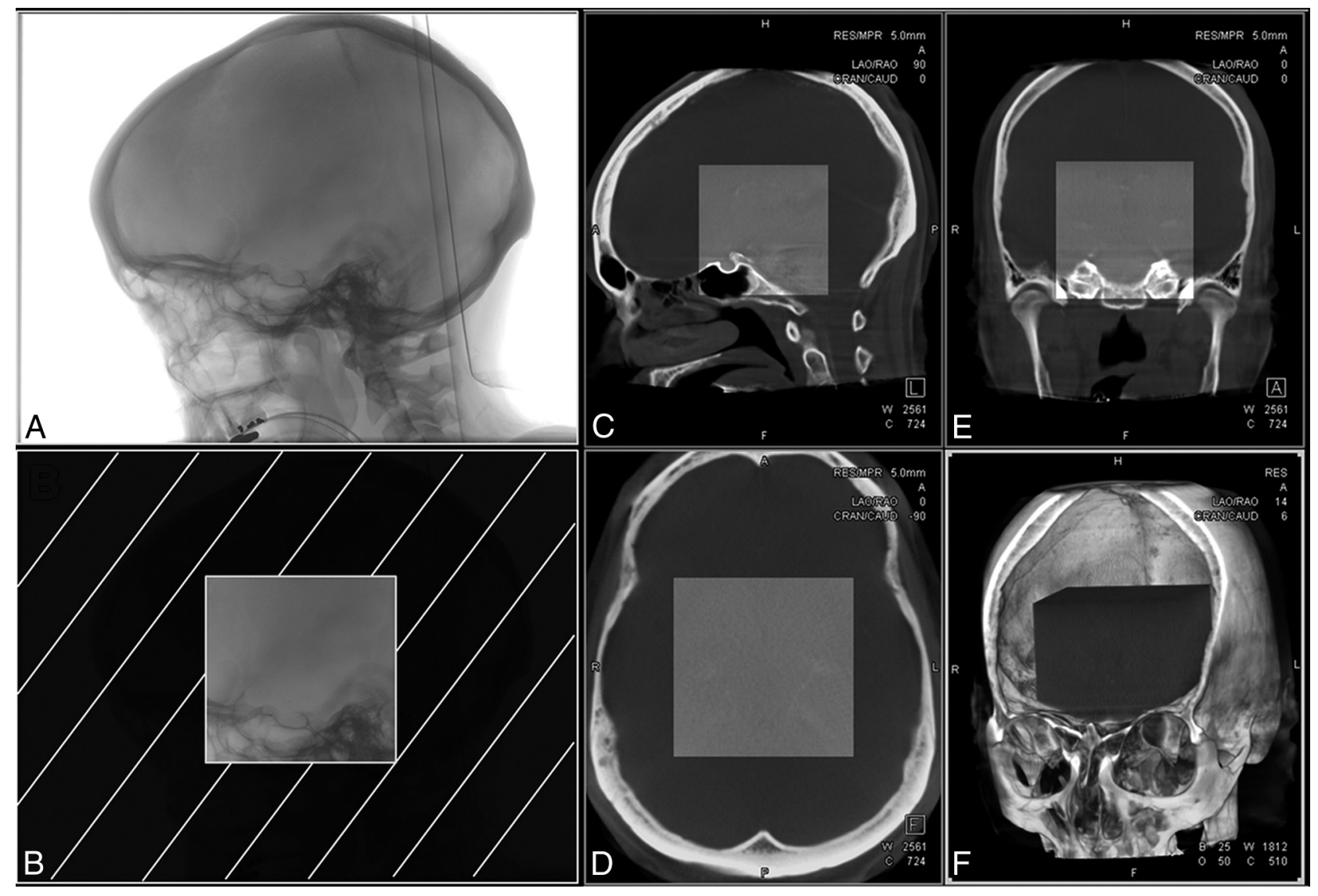

FIG 1. Difference between full FOV CACT and VOI CACT acquisitions. Projection image for FFOV captures of the full head of the patient (A), while the box-collimated VOI scan (B) only exposes and acquires data from a small area of the patient ( $12 \%$ of the FFOV). Triplanar section cuts $(C-E)$ and volumetric illustration $(F)$ demonstrate the fused FFOV CACT and the VOI CACT (central area).

approach yields high-quality 3D images in the volume of interest, while the areas outside the VOI have, because of an increase in noise, reduced image quality. ${ }^{6-8}$ Conventional box-collimated methods have problems stemming from object truncation, usually resulting in compromised image quality of the reconstructions. Attempts to limit truncation artifacts by using mathematic techniques to account for the missing data have had limited success, with the postprocessed image quality still being inferior to reconstructions in which truncation was not present. ${ }^{9-13}$

Recently, however, a newly introduced method has yielded results in which image quality of a device inside a tightly collimated VOI differs little, if at all, from reconstructions made by using nontruncated VOI acquisitions. ${ }^{14,15}$ In this study, we investigated the radiation dose reduction achieved by using the technique and the potential benefits that such images might provide during endovascular treatment of intracranial aneurysms with flow diverters or stent-assisted coiling.

\section{MATERIALS AND METHODS}

\section{Patient Selection}

Twenty-seven consecutive patients having VOI CACT scans were identified from an institutional review board-approved data base. VOI CACT was routinely performed after flowdiverter placement and often after stent placement for stentassisted coiling of aneurysms. Our sample includes a heterogeneous population of patients with variations in aneurysm locations, vessel sizes, pathologies, and device types. These reflect the distribution of pathologies seen during this interval. Most pathologies were aneurysms $(25 / 27,92.6 \%)$ followed by stenosis $(2 / 27,7.4 \%)$. Included are 21 saccular aneurysms $(77.8 \%)$ and 4 fusiform aneurysms (14.8\%). Of the 25 aneurysms, $10(40 \%)$ were treated with conventional stent-assisted coiling and $15(60 \%)$ were treated with flow diversion. All aneurysms in this series were unruptured. The devices used were either the Neuroform EZ Stent System (Stryker Neurovascular, Kalamazoo, Michigan), the Enterprise self-expanding stent (Codman \& Shurtleff, Raynham, Massachusetts), or the Pipeline Embolization Device (Covidien, Irvine, California).

\section{Data Acquisition}

A VOI acquisition was performed as desired by the treating physician during an intervention (eg, post-flow-diverter placement or end point of treatment). In all acquired datasets, the patient was positioned in a head-first, supine position and the injection catheter was placed at the origin of the artery supplying the vascular structure that had been treated. The volume-of-interest scan is a variation of a high-quality CACT (DynaCT; Siemens, Erlangen, Germany), modified by introducing lateral and vertical collimation, effectively reducing the image FOV by either $28 \%$ or $12 \%$ (Fig 1).

For our study, the $12 \%$ collimation mode was used exclusively, with collimators placed automatically by the system on selection 
of the VOI examination protocol by the operator. The contrastinjection protocol was $40 \mathrm{~mL}$ of iohexol (Omnipaque, $300 \mathrm{mg} / \mathrm{L}$ iodine; GE Healthcare, Piscataway, New Jersey) diluted to between $20 \%$ and $50 \%$ and injected at the rate of $2 \mathrm{~mL} / \mathrm{s}$ with an $\mathrm{x}$-ray delay of 2 seconds. From experience, this protocol is known to provide good visualization of the vascular structures without obscuring visualization of an implanted device. The acquisition parameters for the VOI acquisitions were $70 \mathrm{kV}$ (peak) and 1.2 $\mu \mathrm{Gy} /$ Frame. The acquisition parameters for the 30 previously acquired full FOV (FFOV) DynaCTs were identical to these factors.

\section{Image Postprocessing}

Reconstructing truncated projection data by using conventional projection image preprocessing and a Feldkamp back-projection algorithm results in an incorrect Hounsfield unit and a bright ring with a broad halo at the boundary of the reconstructed image. To avoid these artifacts, the VOI technique uses the approximate, truncation-robust algorithm for CT, which replaces the projection image-preprocessing step used in the conventional reconstruction (the main contributor to the aforementioned image artifacts) with a Laplacian derivative filter followed by a nonlocal 2D residual filter. ${ }^{14,15}$ The Laplacian derivative filter minimizes the signal drop at the edges of the recorded images, which is the main contributor to image artifacts. Volumetric images reconstructed from truncated data by using this method yield highly similar characteristics and image content (ie, image quality) compared with nontruncated acquisitions. ${ }^{14}$ All acquired datasets were retrospectively processed by using a prototype software implementation of this algorithm.

\section{Dose Measurement}

Common $\mathrm{x}$-ray radiation measures reported from examinations performed in the angiography suite are dose-area product (milligray $\times$ square centimeter), a product of the radiation dose measured at the $\mathrm{x}$-ray tube exit window and the exposed area, and skin exposure (milligray), an estimate based on standard calibration measurements and system position. These static measurements do not yield a good understanding of how the radiation dose to the patient is distributed during a rotational scan acquisition. To supplement this information and to gain a better understanding of the dose distribution during a VOI CACT acquisition, for our study, we used an anthropomorphic head phantom and thermoluminescence dosimetries (TLDs).

The head phantom consisted of a human skull embedded in tissue-mimicking material (Rando Head Phantom; The Phantom Laboratory, Salem, New York) with evenly distributed bore holes allowing the placement of radiation detectors. TLDs are radiation-sensitive chips ( $1 \mathrm{~cm}$ in diameter, $0.5 \mathrm{~mm}$ high) that emit stored ionizing radiation as light when heated. Thirty-five TLDs were placed symmetrically throughout the phantom. The phantom was then placed on the table of a clinical biplane C-arm system (DynaCT; Siemens), and independent measurements were made for the conventional full FOV DynaCT rotational acquisitions and a $12 \%$ square collimated VOI mode that was identical to the VOI acquisition mode used for the clinical subjects. Acquisition parameters were set to the following: $200^{\circ}$ scan range, 496 projection images, $70 \mathrm{kVp}$, and $1.2 \mu \mathrm{Gy} /$ Frame. For the phantom studies, 3 rotational acquisitions were performed in an effort to minimize errors resulting from possible $\mathrm{x}$-ray tube output fluctuations. The exposed TLDs were evaluated by an accredited laboratory (Calibration Lab, Madison, Wisconsin). These TLD results were read in custom-designed software and interpolated across the phantom by using a second-order polynomial function. While this first-order approximation cannot fully account for the attenuation and scatter characteristics of different tissues, it gives the observer a good representation of the distribution of the radiation exposure. ${ }^{16}$ Finally, the dose-area products of 30 consecutive full field of view DynaCTs were recorded and used for comparison with the dose-area products of the VOI acquisitions.

\section{Image Evaluation}

Three experienced neuroendovascular surgeons independently performed a subjective evaluation of the VOI images. For each case, the reviewers were given images from both volume-rendered and multiplanar-rendered reconstructions and a video showing manipulation of the $3 \mathrm{D}$ volumes in a way that best demonstrated the device and its relationship with the relevant vasculature. All the reviewers were asked to complete an evaluation form assessing the following details by using a 2-point rating scale (ie, yes or no): Did the VOI CACT images determine whether the device was completely open, the apposition of the device to the arterial wall, the position of the device related to the aneurysm ostium, visualization of the entire device configuration after deployment, and clear visualization of the arteries of interest? No other clinical data were provided to the reviewers.

\section{Statistics}

The statistical analysis was performed by using SPSS 20.0 (IBM, Armonk, New York). The qualitative evaluation data were presented as percentages. Intraclass correlation was performed, and Cronbach $\alpha$ coefficients were calculated to evaluate the interobserver consistency for each qualitative variable. The interobserver consistencies were described as unacceptable $(\alpha<.5)$, poor $(.5 \leq$ $\alpha<.6)$, acceptable $(.6 \leq \alpha<.7)$, good $(.7 \leq \alpha<.9)$, and excellent $(\alpha \geq .9)$.

\section{RESULTS}

\section{Dose Measurement}

TLD radiation dose measurements yielded an average dose of 74.5 mGy (median, 68.8 mGy; range, 32.5-179.9 mGy) for full FOV DynaCT and $15.4 \mathrm{mGy}$ (median, 7.9 mGy; range, 2.1-54.5 mGy) for VOI CACT. An evaluation of dose distribution across identical locations showed a dose reduction of $\sim 40 \%$ at the back of the head ( $\mathrm{x}$-ray tube rotating under the table) and up to $90 \%$ at the front of the head (Fig 2). The average reduction in radiation dose was $79.8 \%$ (median, $88.9 \%$; range, 36.5\%-97.5\%). A comparison of dose-area product measurements as reported for the 30 previously acquired full FOV DynaCT studies with dose-area product measured in the VOI acquisitions showed a similar reduction of $\sim 85 \%$ for the VOI studies. From our results, the average radiation dose-area product of a VOI acquisition recorded at the tube exit window was $1140 \mu \mathrm{Gy} \times \mathrm{m}^{2}$; this is equivalent to that of a single 10 -second biplane 2D DSA angiogram. ${ }^{5}$ 


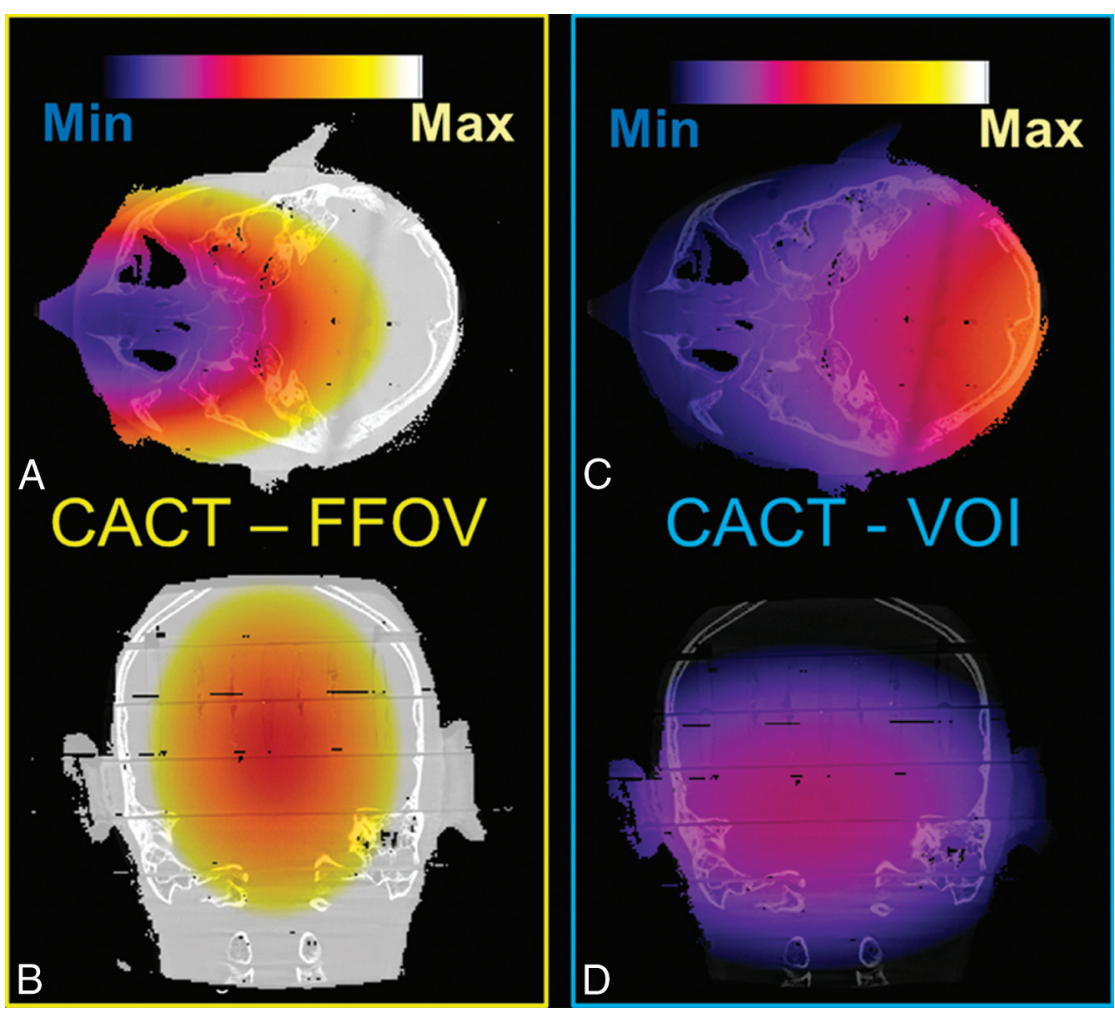

FIG 2. Comparison of dose distribution between FFOV CACT ( $A$ and $B)$ and VOI CACT with an exposure area of $12 \%$ of the FFOV acquisition $(C$ and $D$ ). All images are displayed on the same scale, normalized to the maximum radiation dose measured in the FFOV CACT image. The shape of the dose distribution is similar between both modes, with substantial reduction in the dose for the VOI CACT, especially at the front of the head. Min indicates minimum; Max, maximum.

Results of qualitative evaluation of VOI CACT images from 3 independent observers

\begin{tabular}{lrrrc}
\hline \multicolumn{1}{c}{ Evaluating Details } & Observer 1 & Observer 2 & Observer 3 & $\begin{array}{c}\text { Cronbach } \\
\boldsymbol{\alpha}\end{array}$ \\
\hline Clinically useful information gained & $24 / 27,88.9 \%$ & $24 / 27,88.9 \%$ & $25 / 27,92.6 \%$ & .810 \\
Determination of stent opening & $25 / 27,92.6 \%$ & $19 / 27,70.4 \%$ & $23 / 27,85.2 \%$ & .628 \\
Determination of stent apposition & $23 / 27,85.2 \%$ & $19 / 27,70.4 \%$ & $21 / 27,77.8 \%$ & .766 \\
Determination of stent position & $18 / 27,66.7 \%$ & $18 / 27,66.7 \%$ & $26 / 27,96.3 \%$ & .601 \\
Good visualization of entire stent & $25 / 27,92.6 \%$ & $21 / 27,77.8 \%$ & $22 / 27,81.5 \%$ & .734 \\
$\begin{array}{l}\text { Good visualization of arteries of } \\
\text { interest }\end{array}$ & $25 / 27,92.6 \%$ & $17 / 27,63.0 \%$ & $21 / 27,77.8 \%$ & .761 \\
\hline
\end{tabular}
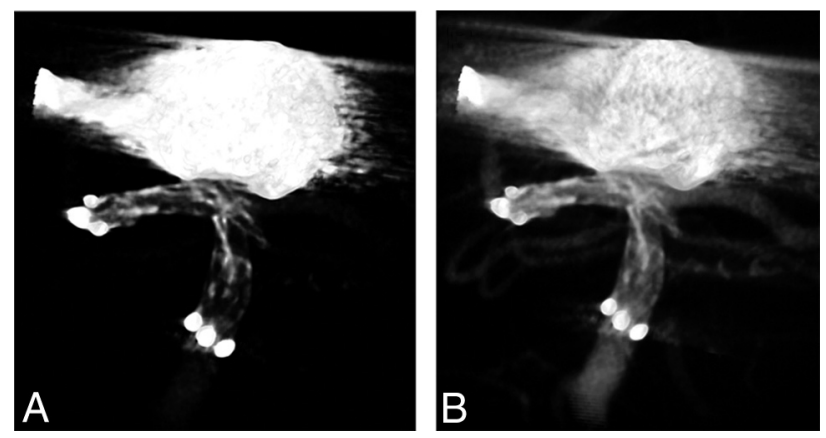

FIG 3. A volume-rendering image $(A)$ shows the open cell design of a Neuroform EZ stent for the treatment of a basilar tip aneurysm. Another image with a different window level $(B)$ shows the stent struts protruding into the left superior cerebellar artery.

\section{Qualitative Image Evaluation}

The qualitative evaluation results of all 3 independent observers and statistical results of intraclass correlations are presented in the Table. For all 6 evaluated variables, acceptable-to-excellent interobserver agreements were observed. For most cases $(88.9 \%-92.6 \%), 3$ observers were in good agreement $(\alpha=.810)$ that VOI CACT was clinically useful.

\section{DISCUSSION}

In this small series, we have shown that the VOI can provide information that is not available from conventional 2D DSA and fluoroscopy, such as full stent deployment, good wall apposition, optimal stent position, and full ostium coverage. Such information may impact the safety and efficacy of stent-assisted coiling. It thus seems likely that the content of VOI images may influence treatment decisions both during and after an intervention (eg, posttreatment anticoagulation regimen, balloon dilation of a stent, placement of a second stent, and so forth). We have also demonstrated that this information may be achieved by using a dramatically reduced radiation dose compared with conventional full FOV CACT imaging.

\section{Clinical Use of VOI}

In our study, independent observer evaluations showed good agreement among 3 observers on the ability of VOI to visualize the fine details of a device and its relationships to a lesion and the adjacent vasculature. These details include the following: First, device configurations such as pore distribution; cell type (which makes the identification of different stents possible); and degree of stent opening, kinking, and fracture (Fig 3). Before the advent of CACT, information on how stent struts are configured within a stent could only be inferred indirectly (eg, from the deflections of a microguidewire as it was advanced through the device). Soon after the introduction of CACT, reports appeared showing the ability of this technology to create highspatial-resolution images of these high-contrast objects. ${ }^{17}$ With VOI CACT, microstructural changes of devices can be visualized, allowing a more complete understanding of these variations in stent configurations. The use of contrast in conjunction with a VOI acquisition allows visualization of these features and their relationship to the lesion being treated and the adjacent vasculature. However, it is still unclear how this information would affect posttreatment antiplatelet and anticoagulation regimens. Second, these details also include the relationships between a device and a vessel, such as the apposition of the stent to the vessel wall, and its positioning relative to an aneurysm ostium. These features were also clearly seen with this technique. Although 2D angiography 

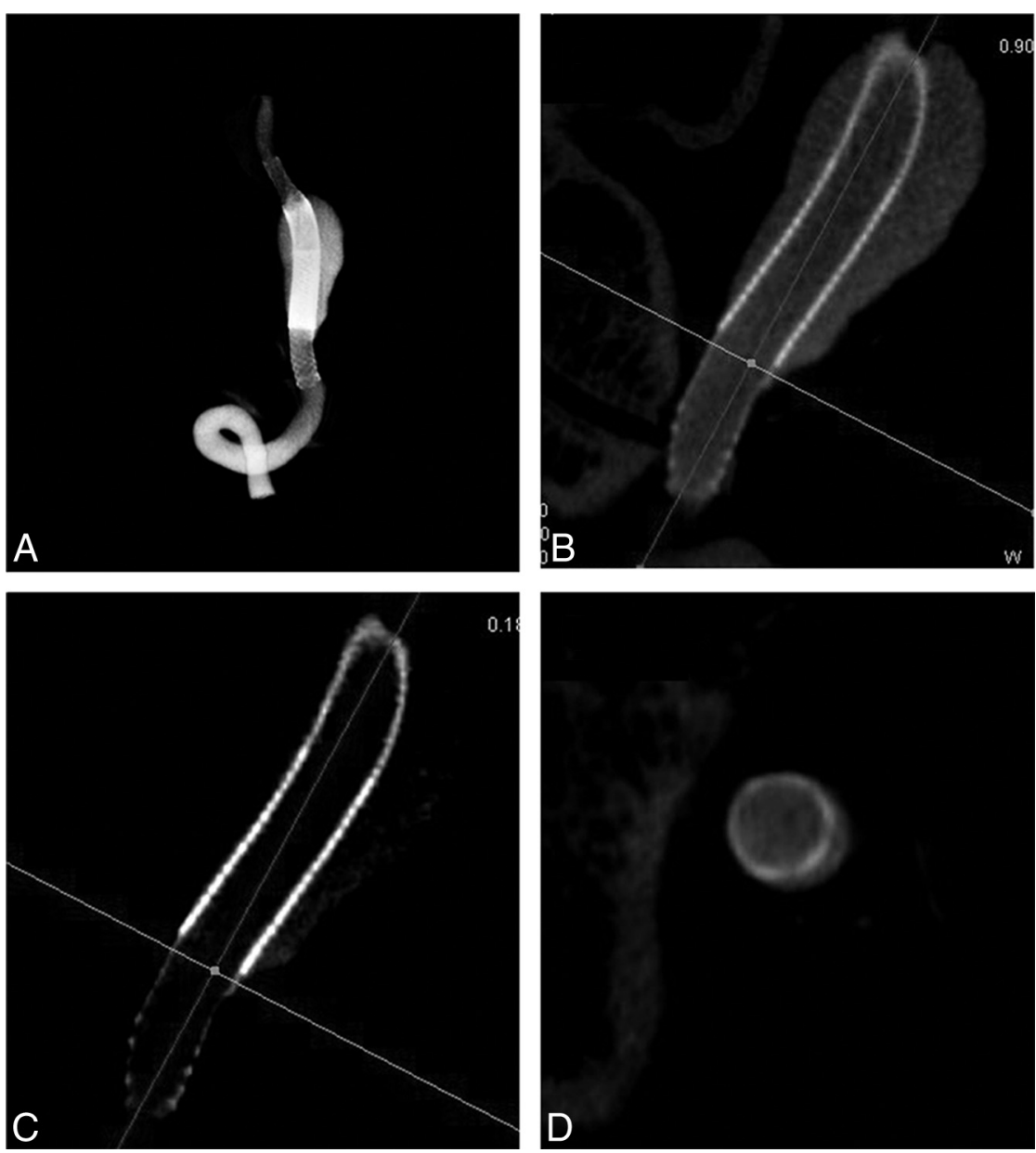

FIG 4. A volume-rendering image $(A)$ shows double flow diverters (Pipeline Embolization Device) for the treatment of a fusiform aneurysm located at the V4 segment of the vertebral artery. Horizontal brighter lines in images of different window levels $(B$ and $C$ ) indicate the most proximal part of aneurysm, which is not covered by double Pipeline Embolization Devices. The corresponding axial view $(D)$ clearly shows that the aneurysm is not fully covered by double layers, which may indicate the malposition of the second FD and require further adjustment or implanting another FD.

and fluoroscopy provide some information about these features, in our experience, the accuracy and ease of stent visualization is not nearly as good as that achieved with VOI CACT (Figs 4 and 5).

VOI CACT performed in conjunction with diluted contrast also provides fine details about both a Pipeline Embolization Device mesh (ie, telescoped or stretched) and a Pipeline Embolization Device or stent relationship with an aneurysm ostium (Fig 4). According to previous reports, ${ }^{18,19}$ the actual porosity of the flow diverter (FD) may be calculated or closely approximated on the basis of the number of the intersections, the angle between the filaments, and the known filament width. This information may potentially be very useful for predicting the angiographic outcome of the treated aneurysms and by providing real parameters for computational fluid dynamics simulations. However, this application will be limited in instances in which there is either the use of multiple FDs or FDs and coils.

Previous studies have shown that image quality of VOI CACT may be further improved, compared with conventional CACT, ${ }^{6,7}$ due to the inherent reduction of scatter radiation achieved by collimation. However, the image quality of VOI CACT can also be degraded because of other factors. For example, beam-hardening artifacts may arise because of such factors as insufficiently diluted contrast medium or the presence of platinum coils and platinum marker tips on a microcatheter or stent. Theoretically, the contrast concentration used should be as low as possible while still providing adequate opacification of the vessel. Because the elements of different devices have different radio-opacities, the optimal contrast concentration will vary somewhat from case to case; in our study, the contrast concentration ranged from $20 \%$ to $50 \%$. Pipeline Embolization Devices and stents used for stentassisted coiling cause few artifacts, whereas platinum coils, depending on the packing density, may cause severe degradation of the image quality. Nonetheless, depending on the aneurysm morphology, the packing density and, most important, the orientation of the aneurysm to the stent, clinically useful information may still often be obtained, even in the presence of these artifacts. To minimize artifacts of this nature, metal artifact-reduction techniques can be used. ${ }^{20}$ In our study, one of our limitations was that we did not evaluate the use of these algorithms. Another limitation for understanding the full clinical utility of this technique is that with currently available stents, full deployment can only be obtained after detachment from the delivery system. This drawback only allowed us to perform a retrospective (eg, postdeployment) analysis rather than use VOI to assess positioning and deployment before a decision had been reached to implant a device. As more devices become available that may be fully deployed before detachment, we expect the VOI to assume an even more important role in monitoring of interventions.

\section{Radiation Dose Reduction}

Earlier studies have shown the potential of using VOI CACT as an effective radiation-saving technique in clinical applications-for example, imaging high-contrast osseous structures such as the inner ear ${ }^{21,22}$ and implants such as intracranial stents. Although these previous studies have shown dramatic reduction of the radiation dose by using VOI CACT, the diagnostic utility and dose reduction have not, to our knowledge, been evaluated in an endovascular clinical practice. Because the radiation dose is directly related to the size of collimated FOV, the dose may be chosen according to the requirements of the scan (ie, it can potentially be further reduced compared with the reduction achieved in this study). The low radiation burden associated with a VOI acquisition, in our opinion, largely removes the limitation of performing multiple acquisitions, as needed, during a procedure. 

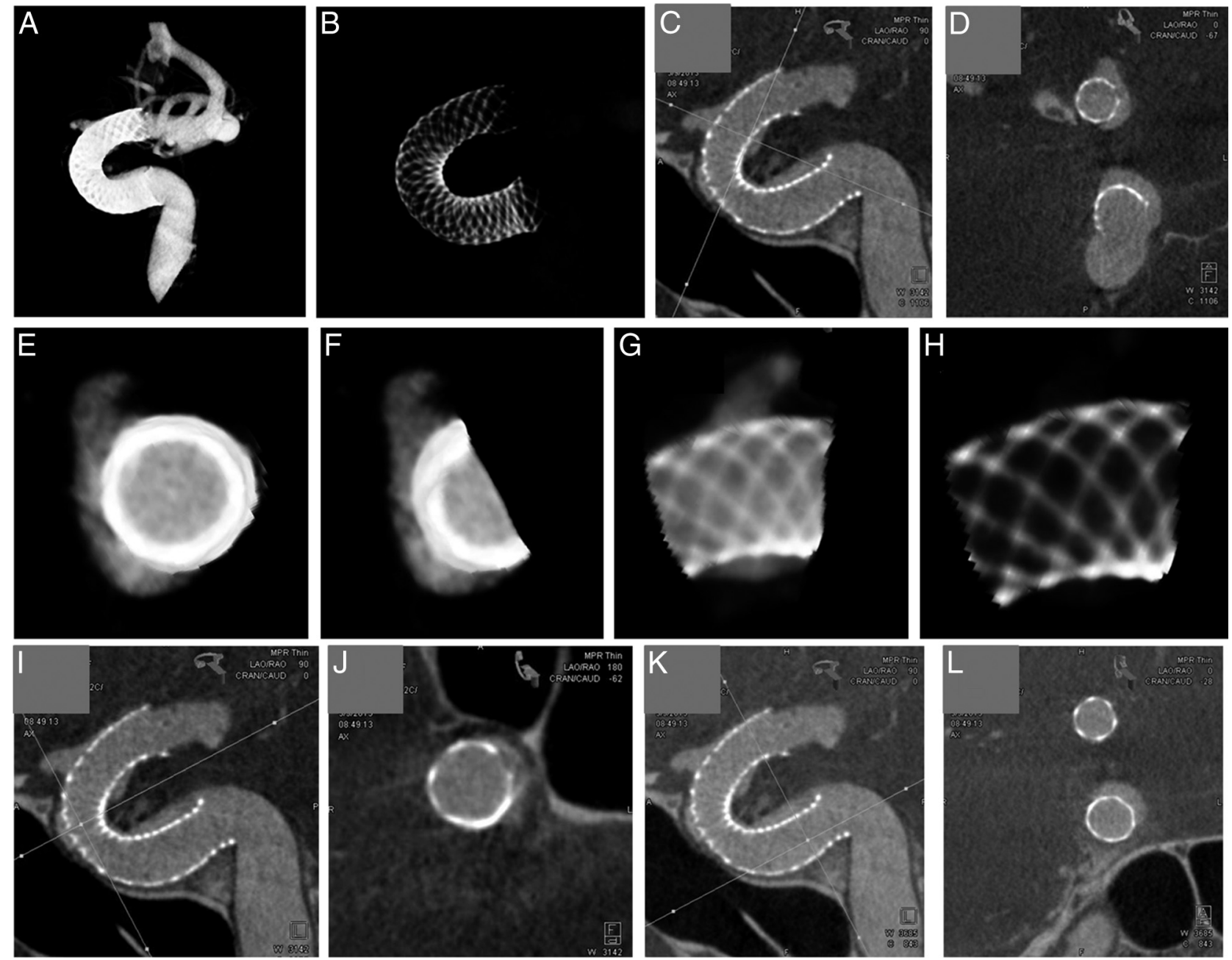

FIG 5. Images $(A$ and $B)$ show a Pipeline Embolization Device for the treatment of an anterior wall aneurysm of the ICA. Horizontal line (C) indicates the position of the image $(D)$, which shows the opening status of the FD and its real coverage of the aneurysm. Another volumerendering image $(E)$ shows the ROI with the aneurysm and FD. After we cut out the contralateral part of the ROI $(F)$, turned approximately $90^{\circ}$ $(G)$, and adjusted the window level $(H)$, the image shows fine details about the mesh, which may facilitate the calculation of the actual porosity of the FD covering the aneurysm. The horizontal line in / and the vertical line in $K$ indicate the position of images J and $L$, respectively, which show the poor apposition of the FD, which may be neglected on traditional 2D angiography or fluoroscopy.

\section{CONCLUSIONS}

The VOI CACT imaging technique offers a dramatic reduction in radiation to the patient while still providing high-quality images of implanted devices. The information provided by these images has clinical significance that may influence treatment during and following an intervention. The dramatic reduction of the radiation dose may allow multiple image acquisitions (if needed) of focused anatomic regions during both critical stages of device deployment and subsequent angiographic follow-up.

Disclosures: Sebastian Schafer-RELATED: Other: employee of Siemens USA. Beverly Aagaard-Kienitz—RELATED: Other: Siemens, ${ }^{*}$ Comments: This was a prototype, so research support regarding this software was provided by Siemens; UNRELATED: Consultancy: Occasionally, I am an unpaid advisor to Siemens. Kevin RoyaltyRELATED: Employment: I am a full-time employee of Siemens USA. Charles Strother-RELATED: Other: Under a master research agreement between Siemens and the University of Wisconsin School of Medicine and Public Health, I receive research support*; UNRELATED: Under a master research agreement between Siemens and the University of Wisconsin School of Medicine and Public Health, I receive research support.* *Money paid to the institution.

\section{REFERENCES}

1. Benndorf G, Strother CM, Claus B, et al. Angiographic CT in cerebrovascular stenting. AJNR Am J Neuroradiol 2005;26:1813-18 Medline

2. Kalender WA, Kyriakou Y. Flat-detector computed tomography (FD-CT). Eur Radiol 2007;17:2767-79 CrossRef Medline

3. Richter G, Engelhorn T, Struffert T, et al. Flat panel detector angiographic CT for stent-assisted coil embolization of broad-based cerebral aneurysms. AJNR Am J Neuroradiol 2007;28:1902-08 CrossRef Medline

4. Kizilkilic O, Kocer N, Metaxas GE, et al. Utility of VasoCT in the treatment of intracranial aneurysm with flow-diverter stents. J Neurosurg 2012;117:45-49 CrossRef Medline

5. Struffert T, Hauer M, Banckwitz R, et al. Effective dose to patient measurements in flat-detector and multislice computed tomography: a comparison of applications in neuroradiology. Eur Radiol 2014;24: 1257-65 CrossRef Medline

6. Schafer S, Noël PB, Walczak AM, et al. Filtered region of interest cone-beam rotational angiography. Med Phys 2010;37:694-703 CrossRef Medline

7. Kolditz D, Kyriakou Y, Kalender WA. Volume-of-interest (VOI) imaging in C-arm flat-detector $\mathrm{CT}$ for high image quality at reduced dose. Med Phys 2010;37:2719-30 CrossRef Medline 
8. Chityala R, Hoffmann KR, Rudin S, et al. Region of interest (ROI) computed tomography (CT): comparison with full field of view (FFOV) and truncated CT for a human head phantom. Proc SPIE Int Soc Opt Eng 2005;5745:583-90 Medline

9. Cho S, Bian J, Pelizzari CA, et al. Region-of-interest image reconstruction in circular cone-beam microCT. Med Phys 2007;34: 4923-33 CrossRef Medline

10. Ohnesorge B, Flohr T, Schwarz K, et al. Efficient correction for CT image artifacts caused by objects extending outside the scan field of view. Med Phys 2000;27:39-46 Medline

11. Van Gompel G, Tisson G, Van Dyck D, et al. A new algorithm for 2D region of interest tomography. Proc SPIE 2004;5370:2105-13 CrossRef

12. Faridani A, Finch DV, Ritman EL, et al. Local tomography II. SIAM J Appl Math 1997;57:1095-127 CrossRef

13. Katsevich A. Cone beam local tomography. SIAM J Appl Math 1999; 59:2224-46 CrossRef

14. Xia Y, Dennerlein F, Bauer S, et al. Scaling calibration in region of interest reconstruction with the 1D and 2D ATRACT algorithm. Int J Comput Assist Radiol Surg 2014;9:345-56 CrossRef Medline

15. Dennerlein F, Maier A. Approximate Truncation Robust Computed Tomography: ATRACT. Phys Med Boil 2013;58:6133-48 CrossRef Medline

16. Schafer S, Nithiananthan S, Mirota DJ, et al. Mobile C-arm conebeam $\mathrm{CT}$ for guidance of spine surgery: image quality, radiation dose, and integration with interventional guidance. Med Phys 2011; 38:4563-74 CrossRef Medline

17. Benndorf G, Klucznik RP, Strother CM. Images in cardiovascular medicine: angiographic computed tomography for imaging of underdeployed intracranial stent. Circulation 2006;114:e499-500 CrossRef Medline

18. Wang K, Yuan S. Actual metal coverage at the neck is critical for flow-diverting stents in treating intracranial aneurysms. AJNR Am J Neuroradiol 2013;34:E31-32 CrossRef Medline

19. Hong B, Wang K, Huang Q, et al. Effects of metal coverage rate of flow diversion device on neointimal growth at side branch ostium and stented artery: an animal experiment in rabbit abdominal aorta. Neuroradiology 2012;54:849-55 CrossRef Medline

20. van der Bom IM, Hou SY, Puri AS, et al. Reduction of coil mass artifacts in high-resolution flat detector conebeam CT of cerebral stent-assisted coiling. AJNR Am J Neuroradiol 2013;34:2163-70 CrossRef Medline

21. Kolditz D, Struffert T, Kyriakou Y, et al. Volume-of-interest imaging of the inner ear in a human temporal bone specimen using a robotdriven C-arm flat panel detector CT system. AJNR Am J Neuroradiol 2012;33:E124-28 CrossRef Medline

22. Shen Y, Yi Y, Zhong Y, et al. High resolution dual detector volumeof-interest cone beam breast CT: demonstration with a bench top system. Med Phys 2011;38:6429-42 CrossRef Medline 